\title{
Impact loading of square plates with piece wise constant thickness
}

\author{
J. Lellep \& A. Mürk \\ Institute of Mathematics, University of Tartu, Estonia
}

\begin{abstract}
Plastic behaviour of square plates subjected to impact loading is studied. It is assumed that the plates are formed from concentric squares with piece wise constant thickness. An approximate method of mode form motions resorting to the energy balance is used in order to predict maximal residual transverse deflections for plates made of Johansen's material. Numerical results are presented for plates with two different thicknesses.
\end{abstract}

Keywords: square plate, impact loading, inelastic material, optimization.

\section{Introduction}

The problems of dynamic behaviour of structural elements have become of increasing interest in recent years. The dynamic plastic behaviour of beams and axisymmetric plates was investigated by many authors. Reviews of these papers are presented by Jones [1], Yu and Chen [2], Stronge and Yu [3]. However, the only exact theoretical solution on dynamic response of a non-axisymmetric plastic plate is obtained by Cox and Morland [4] who investigated within the framework of thin plate theory the behaviour of square plates subjected to a rectangular pressure pulse.

Jones [1], Cox and Morland [4] and other authors have studied square plates of constant thickness made of a Johansen's material. Blast loaded square plates are investigated by Olson et al. [5]. An approximate technique was developed by Baker [6]. Zhu [7] obtained both, theoretical numerical predictions and experimental results for transient deformation modes of square plates subjected to explosive loadings. It was observed that numerical predictions were in a good agreement with experimental results. 
The phenomenon of saturated impulse in elastic-plastic square plates was investigated by $\mathrm{Zhu}$ and $\mathrm{Yu}[8]$ in the case of a fully clamped plate.

Lellep and Mürk [9, 10] presented a method for determination of residual deflections of stepped annular and square plates subjected to impulsive loadings.

Approximate procedures for investigation of rigid-plastic rectangular plates subjected to dynamic loadings are developed by Jones et al. [11, 12], Yu and Chen [13]. Theoretical predictions suggested by Jones and Symonds give surprisingly good agreement with corresponding experimental results.

In the present paper this approach is used in the case of small deflections of stepped square plates subjected to impact and impulsive loadings.

\section{Formulation of the problem}

In what follows, the behaviour of fully plastic plates will be studied in the framework of the classical theory of thin plates and the concept of a rigid-plastic body.

Let us consider a square plate with a side length $2 L$ (fig. 1$)$. The plate under consideration is subjected to the initial impact loading.

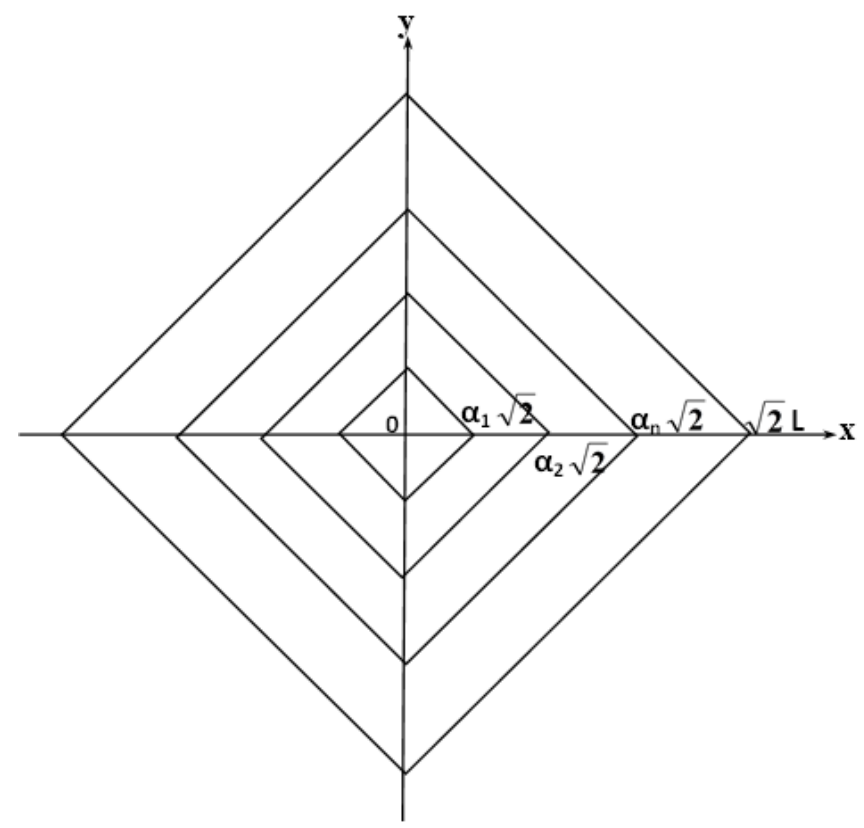

Figure 1: A square plate.

In the following we shall consider the cases when at the initial moment $t=0$ $p>0$ and when $p=0$ (now the motion of the plate is caused by the inertia).

In the latter case we assume that the initial kinetic energy $K_{0}$ is given whereas the initial transverse velocity field $v_{0}(x, y)$ may be unknown. It is assumed herein 
that the origin of the coordinate system Oxy is placed at the centre of the plate and the axes are directed towards diagonals of squares (fig. 1).

We are considering plates with piece wise constant thickness. Thus it is assumed that

$$
h=h_{j}
$$

for $(x, y) \in \bar{D}_{j} ; j=0, \ldots, n$. We restrict our attention to the concentric case when the inner and outer boundaries of regions $\bar{D}_{j}$ are squares. Let the boundaries of regions of constant thickness intersect $x$ - and $y$ - axis at points $0, \alpha_{1} \sqrt{2} L, \ldots$, $\alpha_{n} \sqrt{2} L, \sqrt{2} L$. Here $\alpha_{0}=0, \alpha_{n+1}=1$.

It is well known in the engineering and fracture mechanics that defects like flaws, notches, cracks are probably unavoidable during manufacturing as well as under repeated loading of structural elements.

It is assumed herein that the plate under consideration has cracks at re-entrant corners of steps. Let $c_{j}$ be the length (deepness) of the straight crack located at the inner boundary of the region $D_{j}$. We assume that the cracks are stable part through surface cracks. The development and propagation of cracks is outside the scope of current paper.

The aim of the paper is to assess the maximal deflections of the stepped plate subjected to the initial impact or impulsive loading.

\section{Governing equations}

According to the basic hypotheses of the concept of a rigid- plastic body it is assumed that elastic stains are small in comparison with plastic strains so that elastic counterparts of strains can be neglected. The material of plates is an isotropic homogeneous material which can be treated as an ideal plastic material obeying Johansen's yield condition given by equalities

$$
\left|M_{1}\right| \leq M^{0}
$$

and

$$
\left|M_{2}\right| \leq M^{0},
$$

where $M_{1}, M_{2}$ stand for principal moments and $M^{0}$ is the yield moment. The moments $M_{x}, M_{y}, M_{x y}$ with shear forces $Q_{x}, Q_{y}$ have to satisfy equilibrium equations

$$
\begin{aligned}
& \frac{\partial Q_{x}}{\partial x}+\frac{\partial Q_{y}}{\partial y}+p=\mu h \frac{\partial^{2} W}{\partial t^{2}} \\
& \frac{\partial M_{x}}{\partial x}+\frac{\partial M_{x y}}{\partial y}-Q_{x}=0 \\
& \frac{\partial M_{y}}{\partial y}+\frac{\partial M_{x y}}{\partial x}-Q_{y}=0
\end{aligned}
$$


Here $\mu$ stands for the material density, $p$ is the intensity of transverse loading and $W$ is the transverse deflection. If the motion of the plate is due to inertia we can take $p=0$. It is well known that the curvatures corresponding to (3) have the form

$$
\kappa_{x}=-\frac{\partial^{2} W}{\partial x^{2}}, \kappa_{y}=-\frac{\partial^{2} W}{\partial y^{2}}, \kappa_{x y}=-\frac{\partial^{2} W}{\partial x \partial y}
$$

\section{Integration of governing equations and residual deflections}

We assume that the deformation process is symmetrical with respect to coordinate axes. Due to the symmetry we can restrict our attention to the first quadrant only. Following Cox and Morland [4] we introduce a new variable

$$
z=\frac{1}{\sqrt{2} L}(x+y)
$$

Evidently, in the first quadrant $0 \leq z \leq 1$. The method of mode form motions will be used in the present paper. This method was suggested by Martin and Symonds (see Symonds [14]). It was established that the approximate theoretical predictions are in good correlation with exact solutions and experimental results (see Baker [6], Jones [1]).

Perhaps the simplest kinematically admissible transverse velocity distribution is

$$
\frac{\partial W}{\partial t}=\dot{W}_{0}(t)(1-z)
$$

where $W_{0}(t)$ stands for an unknown function of time. Evidently, $\dot{W}_{0}(t)$ is the transverse velocity of the central point of the plate.

Eliminating of shear forces $Q_{x}, Q_{y}$ from (3) and taking (5), (6) into account leads to the equation

$$
\frac{\partial^{2} M_{x}}{\partial x^{2}}+2 \frac{\partial^{2} M_{x y}}{\partial x \partial y}+\frac{\partial^{2} M_{y}}{\partial y^{2}}=\mu h_{j} \ddot{W}_{0}(1-z)-p
$$

which holds good in the region $D_{j}(j=0, \ldots, n)$. Here we interpret $D_{j}$ as the part of the region $\bar{D}_{j}$ which belongs to the first quadrant only where $z \in\left(\alpha_{j}, \alpha_{j+1}\right)$. 
In (7) $\ddot{W}_{0}$ stands for the acceleration of the central point of the plate at $z=0$. It is assumed to be a function depending on time only. We are looking for the solution of (7) in the form

$$
\begin{aligned}
& M_{x}=M_{j}+x^{2} F_{j}(z), \\
& M_{y}=M_{j}+y^{2} F_{j}(z), \\
& M_{x y}=x y F_{j}(z),
\end{aligned}
$$

for $(x, y) \in D_{j}$ or $z \in\left(\alpha_{j}, \alpha_{j+1}\right)(j=0, \ldots, n)$. Here $M_{j}$ stands for the limit moment for the part $D_{j}$ of the plate. Thus, $M_{j}=\sigma_{0} h_{j}^{2} / 4, \sigma_{0}$ being the yield stress of the material. Functions $F_{j}(j=0, \ldots, n)$ in (8) are unknown functions depending on the variable $z$.

Boundary conditions at the edge can be obtained from the relation at an inclined edge (see Jones [1], Lellep and Mürk [9])

$$
F_{n}(1)=-\frac{M_{n}}{L^{2}}
$$

From mechanical considerations it is evident that $(M)_{n}$ and $(Q)_{n}$ are continuous when crossing the lines $z=\alpha_{j}(j=1, \ldots, n)$. Thus

$$
\begin{gathered}
M_{j-1}+L^{2} \alpha_{j}^{2} F_{j-1}\left(\alpha_{j}-\right)=M_{j}+L^{2} \alpha_{j}^{2} F_{j}\left(\alpha_{j}+\right), \\
F_{j}\left(\alpha_{j}+0\right)-F_{j-1}\left(\alpha_{j}-0\right)=\frac{M_{j-1}-M_{j}}{L^{2} \alpha_{j}^{2}},
\end{gathered}
$$

and

$$
F_{j}^{\prime}\left(\alpha_{j}+0\right)-F_{j-1}^{\prime}\left(\alpha_{j}-0\right)=\frac{3}{L^{2} \alpha_{j}^{3}}\left(M_{j}-M_{j-1}\right)
$$

for each $j=1, \ldots, n$.

Finally one has to check if the stress field is statically admissible at each point of the plate. Since at $z=\alpha_{j}$ a crack with maximal deepness $c_{j}$ is located the maximal bending moment sustained by the plate is $v_{j} M_{j+1}$ where $0<v_{j}<1$.

Substituting (8) in (7) after appropriate transformations one obtains

$$
z^{2} \frac{d^{2} F_{j}}{d z^{2}}+6 z \frac{d F_{j}}{d z}+6 F_{j}=\mu h_{j} \ddot{W}_{0}(t)(1-z)-p
$$

for $z \in\left(\alpha_{j}, \alpha_{j+1}\right) ; j=0, \ldots, n$. 
It is easy to recheck that the last equation has the solution

$$
F_{j}=\frac{\mu h_{j} \ddot{W}_{0}}{12}(2-z)-\frac{p}{6}+\frac{B_{j}}{z^{2}}+\frac{C_{j}}{z^{3}},
$$

where $B_{j}$ and $C_{j}$ are arbitrary constants.

\section{Determinations of the acceleration}

It can be easily shown that the acceleration $\ddot{W}_{0}$ at the centre of the plate is a constant. Thus one can easily integrate the acceleration which results in

$$
\dot{W}_{0}=\ddot{W}_{0} t+\dot{W}_{0}(0)
$$

and

$$
W_{0}(t)=\ddot{W}_{0} \frac{t^{2}}{2}+\dot{W}_{0}(0) t,
$$

where the initial condition $W_{0}(0)=0$ has been taken into account.

It follows from (14), (15) that the maximal residual deflection is

$$
W_{1}=-\frac{\dot{W}_{0}^{2}(0)}{2 \ddot{W}_{0}}
$$

Let us consider the case $p=0$ and $n=1$ in a greater detail. Evidently, in the central region $F_{0}$ must be finite. This yields $B_{0}=C_{0}=0$ and

$$
F_{0}=\mu h_{0} \ddot{W}_{0}(2-z)
$$

Now jump conditions (10), (11) give for a simply supported plate

$$
\begin{aligned}
B_{1} & =\frac{1}{\left(1-\alpha_{1}\right)}\left\{-\frac{1}{12} \mu \ddot{W}_{0} h_{1}+\frac{1}{12} \mu \ddot{W}_{0} \alpha_{1}^{3}\left(2-\alpha_{1}\right)\left(h_{1}-h_{0}\right)\right. \\
& \left.-\frac{1}{L^{2}}\left[M_{0} \alpha_{1}+M_{1}\left(1-\alpha_{1}\right)\right]\right\}, \\
C_{1} & =\frac{1}{\left(1-\alpha_{1}\right)}\left\{\frac{1}{12} \mu \ddot{W}_{0} h_{1} \alpha_{1}-\frac{1}{12} \mu \ddot{W}_{0} \alpha_{1}^{3}\left(2-\alpha_{1}\right)\left(h_{1}-h_{0}\right)+\frac{M_{0} \alpha_{1}}{L^{2}}\right\} .
\end{aligned}
$$

Making use of (9), (13) one obtains the acceleration

$$
\ddot{W}_{0}=\frac{12}{\mu L^{2}} \frac{M_{0} \alpha_{1}+M_{1}\left(1-\alpha_{1}\right)}{\left[\left(h_{1}-h_{0}\right)\left(3 \alpha_{1}^{4}-8 \alpha_{1}^{3}+6 \alpha_{1}^{2}\right)-h_{1}\right]}
$$

for the simply supported plate. 
Substitution of (19) in (16) leads to the residual deflection

$$
W_{1}=\frac{K_{0}}{8\left[M_{0} \alpha_{1}+M_{1}\left(2-\alpha_{1}\right)_{1}\right]}
$$

\section{Numerical results and discussion}

Results of calculations are presented for the case of plates with single step in figs. $2-5$.

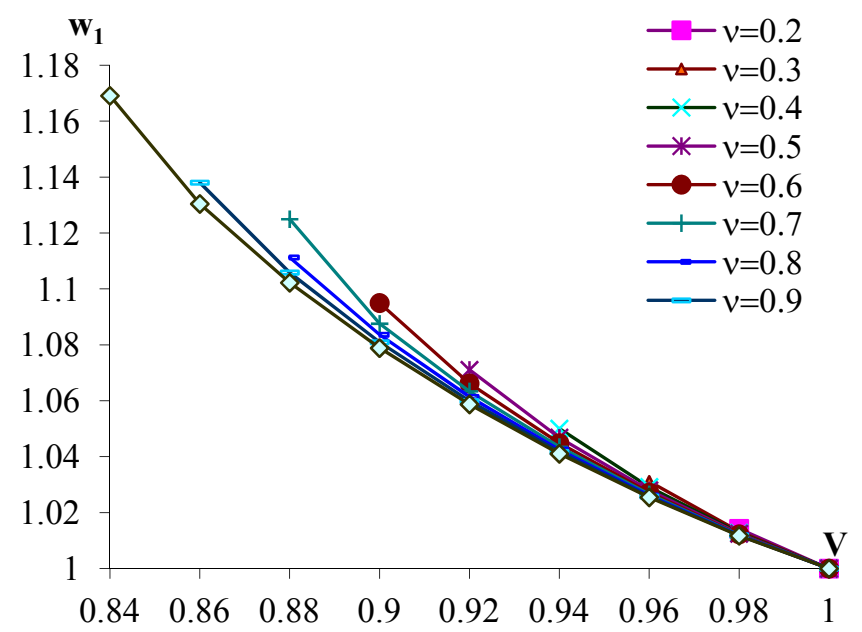

Figure 2: Maximal residual deflections of plates of constant thickness.

Maximal residual deflections $w_{1}$ are presented in figs. 2 and 3 in the case of a square plate subjected to impulsive loading. Here and in the rest illustrations the following notation is used

$$
\begin{aligned}
& w_{1}=\frac{8 M_{0} W_{1}}{K_{0}}, \quad \gamma_{1}=\frac{h_{1}}{h_{0}}, \\
& m_{1,2}=\frac{M_{1,2}}{M_{0}}, \quad V=\frac{\bar{V}}{4 h_{0} L^{2}} .
\end{aligned}
$$

The quantity $\bar{V}$ stands for the volume of the plate. Different curves in fig. 2 are calculated for different values of the crack parameter $v$. Note that $v=1$ in the case of a plate without cracks. Alternatively, if $v=0$, then the crack has penetrated through the plate. It is somewhat surprising that the maximal residual deflection only slightly depends on the crack length (fig. 2). However, the existence of the solution essentially depends on $v$ as it can be seen from fig. 2 . 


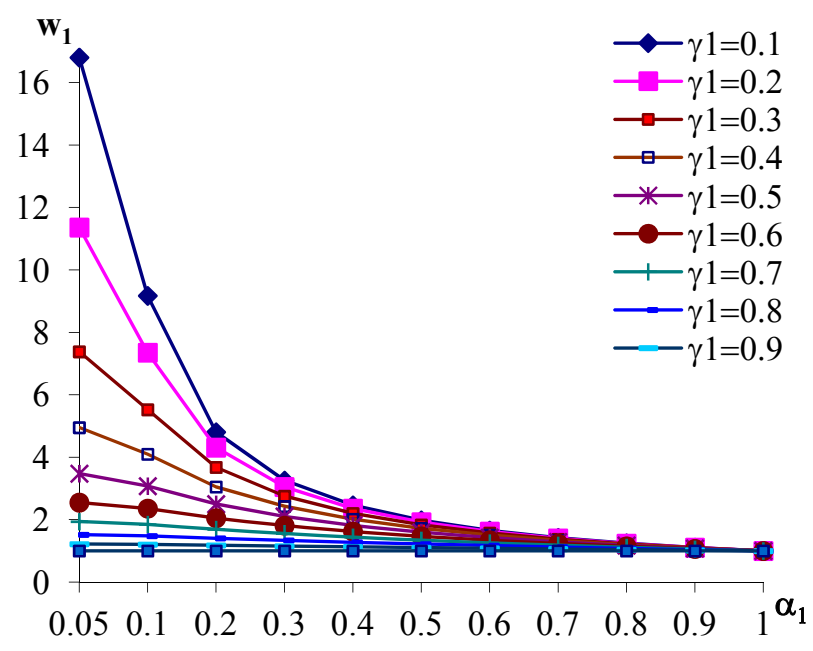

Figure 3: $\quad$ Maximal residual deflections of stepped plates.

Bending moment $m=(M)_{n} / M_{0}$ distributions are presented in fig. 4 . Different curves in Fig. 4 are obtained for different values of the volume parameter $V$. Curves presented in fig. 4 are calculated for the plates without crack (here $v=1$ ).

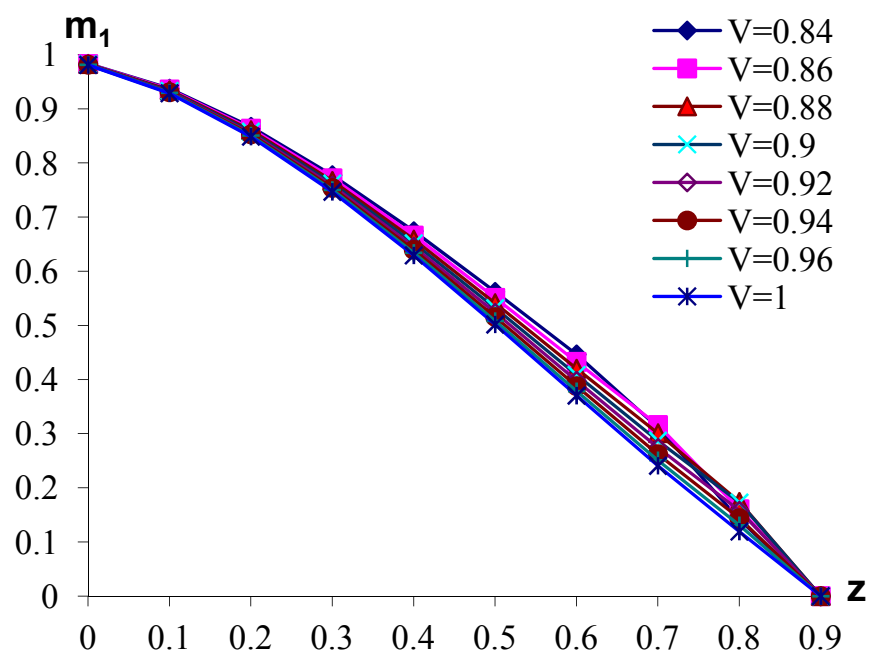

Figure 4: Bending moment $m$ distributions for simply supported plates.

It can be seen from fig. 4 that the bending moment $(M)_{n}$ only slightly depends on the parameter $v$ in the case of simply supported plates. 
The maximal permanent deflection is presented in fig. 5 by line 1 versus

$$
\lambda=\frac{K_{0}}{2 M_{*} h_{*}} \frac{1}{\beta^{2}}
$$

where $\beta=0.593$.

The coefficient $\beta=\frac{B}{L}$ is introduced in order to compare the results obtained by Jones et al. $[11,12]$ for rectangular plates with those corresponding to square plates. The rectangular plates have sizes $2 B$ and $2 L$ whereas $B<L$. It is reasonable to compare permanent transverse deflections of a square plate with transverse deflections of such rectangular plates whose shorter edge is equal to the edge of the square plate.

Curves $2-5$ in fig. 5 present theoretical predictions by Jones et al. [12]; curve 2 corresponds to the case of infinitesimal deflections, curves 3-5 correspond to finite deflection theories with various yield conditions. Curve 3 presents the relationship between transverse deflections and the initial kinetic energy in the case of the maximum normal stress yield condition whereas curves 4 and 5 are associated with circumscribing and inscribing yield criterion, respectively.

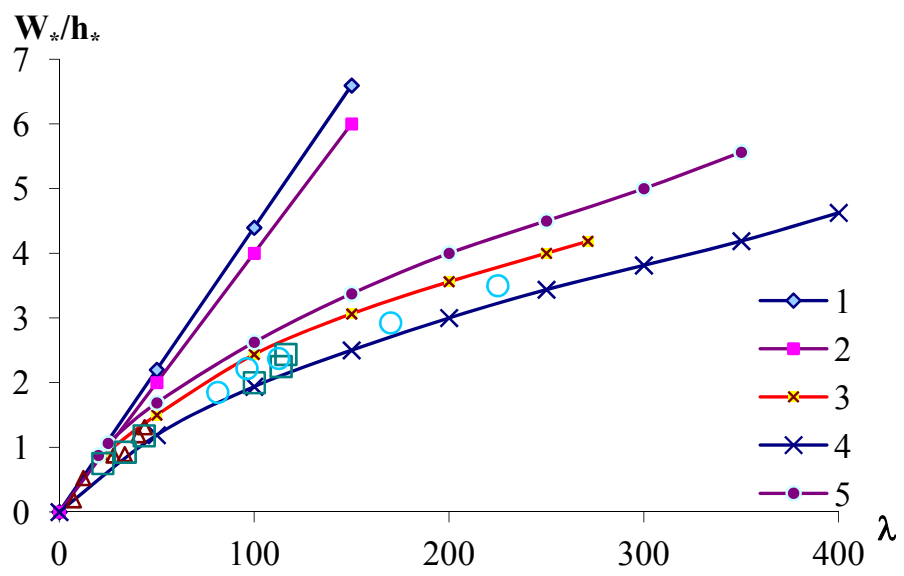

Figure 5: Maximum permanent transverse displacement.

Triangles, squares and circles in fig. 5 denote the experimental results on aluminium 6061-T6 plates obtained by Jones et al. [12] It can be seen from fig. 6 that theoretical predictions suggested in the present paper compare favourably with predictions by Jones for infinitesimal deflections. In the range of small deflections theoretical results are close to the experimental data. The discrepancies of results in the range of large deflections are not surprising as membrance forces are neglected in the current study. 


\section{Concluding remarks}

An approximate method of determination of residual deflections of pure plastic square plates has been developed. The plates have piece wise constant thickness and are subjected to the initial impact loading.

It is assumed that the material of plates obeys Johansen's yield condition and associated flow law. Theoretical predictions are obtained for plates with arbitrary number of concentric steps. The method can be easily extended to the case of blast loaded square plates.

\section{Acknowledgements}

The support from the Grant № 9110 of ESF "Optimization of structural elements" and from the target financed project SF0180081S08 "Models of applied mathematics and mechanics" is acknowledged.

\section{References}

[1] Jones, N., Structural Impact, Cambridge University Press, Cambridge, 1989.

[2] Yu, T.X. and Chen, F.L., The large dynamic plastic response of rectangular [plates. International Journal of Impact Engineering, 12, pp. 603-616, 1992.

[3] Stronge, W. and Yu, T.X., Dynamic Models of Structural Plasticity. Springer Verlag, London, 1993.

[4] Cox, A.D. and Morland L.M., Dynamic plastic deformations of simply supported square plates. Journal of Mechanics and Physics of Solids, 7, pp. 229-241, 1959.

[5] Olson, M., Nurick, G.N. and Fagnan, J.R., Deformation and rupture of blast loaded square plates. Predictions and experiments. International Journal of Impact Engineering, 13, pp. 279-291, 1993.

[6] Baker, W.E., Approximate techniques for plastic deformation of structures under impulsive loading. Shock and Vibration Digest, 7, pp. 107-117, 1975.

[7] Zhu, L., Transient deformation modes of square plates subjected to explosive loadings. International Journal of Solids and Structures, 33, pp. 301-314, 1996.

[8] Zhu, L. and Yu, T.X., Saturated impulsive for pulse-loaded elastic- plastic square plates. International Journal of Solids and Structures, 34, pp. 17091718, 1997.

[9] Lellep, J., Mürk, A., Inelastic behaviour of stepped square plates. In: R. Kienzler, H. Altenbach, I. Ott (eds). Theories of Plates and Shells. Critical Review and New Applications (Euromech Colloquium 444 held in Bremen 2002), Springer, Berlin Heidelberg New York, pp. 133-140, 2004. 
[10] Lellep, J., Mürk, A., Inelastic stepped plates under impulsive loading. In: N.K. Gupta (ed) Plasticity and Impact Mechanics (Implast 2003 held in New Delhi), Phoenix Publishing House, New Delhi, pp. 577-588, 2004.

[11] Jones, N., A theoretical study of the dynamic behaviour of beams and plates with finite deflections. International Journal of Solids and Structures, 7, pp. 1007-1029, 1971.

[12] Jones, N., Uran, T. and Tekin, S.A., The dynamic plastic behaviour of fully clamped rectangular plates. International Journal of Solids and Structures, 6, pp. 1499-1512, 1970.

[13] Yu, T.X. and Chen, F.L., The large dynamic plastic response of rectangular plates. International Journal of Impact Engineering, 12, pp. 603-616, 1992.

[14] Symonds, P.S., Finite elastic and plastic deformations of pulse loaded structures by an extended mode technique. International Journal of Mechanical Sciences, 22, pp. 579-605, 1980. 